\title{
Wind Direction Coding in the Cockroach Escape Response: Winner Does Not Take All
}

\author{
Rafael Levi and Jeffrey M. Camhi \\ Department of Cell and Animal Biology, Life Sciences Institute, Hebrew University, Jerusalem, Israel 91904
}

Cockroaches respond to the approach of a predator by turning away and then running. Three bilateral pairs of giant interneurons are involved in determining the direction of the sensory stimulus and setting the turn direction. Each of these six interneurons has a different directional response to wind stimuli. We have tested whether these six cells use a winner-take-all mechanism to perform this directional determination: that is, each of these cells suppressing the motor response that each of the other cells promotes. Such a mechanism is found in similar behaviors of some other animals. By adding spikes to identified giant interneurons through intracellular stimulation during the sensory-induced behavior and analyzing the resulting directional leg movements, we find that a winner-take-all is not used in this system. Rather, directional determination appears to be based on collaborative calculation of direction by the giant interneurons as a group.

Key words: escape behavior; electrical stimulation; interneurons; giant interneurons; cockroach; winner-take-all; directional behavior; neural code
Many behaviors of animals can be performed in various directions. In some nervous systems, different central neurons are responsible for the different directional forms of a given behavior. Examples include the directionally varied escape behaviors elicited by individual giant interneurons (GIs), in both crayfish and fish (Wine and Krasne, 1972; Eaton et al., 1991), and eye saccades in monkeys, controlled by sets of neurons in the MT cortex (Salzman and Newsome, 1994).

In each of these three examples, the neuron or set of neurons controlling a given directional variant of the behavior suppresses the circuits controlling alternative directions (Roberts, 1968; Krasne and Lee, 1988; Eaton et al., 1991; Salzman and Newsome, 1994). This "winner-take-all" mechanism helps ensure that just one variant of the behavior occurs at any given moment.

In other behaviors, the neurons underlying different behavioral variants do not suppress one another. Rather, they collaborate in an additive manner to produce the behavior. A clear example is population vector computation, which has been shown in the superior colliculus (Sparks et al., 1976), midtemporal (MT) cortex (Groh et al., 1997), and especially the motor cortex (Georgopoulos et al., 1986) to determine motor direction.

We report here on tests for a winner-take-all mechanism underlying the escape behavior of the cockroach Periplaneta americana. Our approach, like that in work on the monkey saccade system (Salzman and Newsome, 1994), was to alter experimentally the activity of the neurons during the behavior and analyze in detail the effect this had on the turn direction. However, here we altered single identified neurons by means of intracellular electrodes, affording much more precise and controlled experi-

\footnotetext{
Received Oct. 18, 1999; revised Jan. 13, 2000; accepted Feb. 11, 2000.

This work was supported by United States of America-Israel Binational Science Foundation Grant 93-00021/3.

Correspondence should be addressed to Jeffrey M. Camhi, Department of Cell and Animal Biology, Hebrew University, Givat-Ram, Jerusalem, 91904 Israel. E-mail: jeff@vms.huji.ac.il.

Dr. Levi's present address: Department of Biology, University of California, San Diego, La Jolla, CA 92093-0357.

Copyright (C) 2000 Society for Neuroscience $\quad 0270-6474 / 00 / 203814-08 \$ 15.00 / 0$
}

mental manipulation than is possible in cortex (Rose et al., 1988; Liebenthal et al., 1994; Gras and Kohstall, 1998; Lewis and Kristan, 1998).

We delivered wind puffs from a given direction, mimicking the natural stimulus that evokes cockroach escape: air displacement produced by the approach of a predator. Simultaneously, we delivered trains of electrical stimuli to identified giant interneurons that are excited by the cockroach's wind sensory cells and that in turn excite the motor circuits of the legs. Because cockroaches tend to turn away from a wind stimulus, a variety of different turn directions can be made, depending on the wind direction. By adding different numbers of spikes to a giant interneuron on different trials, we were able to alter the cockroach's turn direction. We determined whether the resultant alteration was proportionate to the number of spikes we added or showed sudden jumps from one turn direction to another, as expected in a winner-take-all system. In several different tests, we found the change to be proportional, thus ruling out a winner-take-all mechanism in this escape system. Indeed, the GIs appear to collaborate, not compete, in their activation of motor circuits to determine the turn direction.

\section{MATERIALS AND METHODS}

We used adult male cockroaches, Periplaneta americana, in all experiments. We raised the cockroaches at $26^{\circ} \mathrm{C}$, on a $12 \mathrm{hr}$ light/dark cycle, in 50 gallon screen-topped cages and fed them rat chow and water ad libitum.

We monitored the escape direction of tethered cockroaches using a system described previously (Liebenthal et al., 1994; Kolton and Camhi, 1995). Briefly, we tethered the cockroach by the abdomen over a slick surface (glass coated with a film of mineral oil) that permitted it to make normal leg movements in place (Camhi and Levy, 1988) (Fig. 1). The sensory stimulus that induces the escape turn and run is the air displacement that results from a predator's approach (Camhi and Tom, 1978). To evoke this behavior, we delivered controlled wind puff stimuli from different azimuthal directions (peak wind speed, $1.2 \mathrm{~m} / \mathrm{sec}$; time-to-peak, $150 \mathrm{msec})$ from a tube $(40 \mathrm{~mm}$ inner diameter) whose tip was located 60 $\mathrm{mm}$ from the wind receptor organs, the posteriorly located cerci. The wind was produced by a $10 \mathrm{inch}, 100 \mathrm{~W}$ speaker. With the front of the animal designated as $0^{\circ}$ and its hind end as $180^{\circ}$, we delivered wind puffs from $10^{\circ}$ to $150^{\circ}$ on the right $(\mathrm{R})$ side only, at $20^{\circ}$ intervals. 


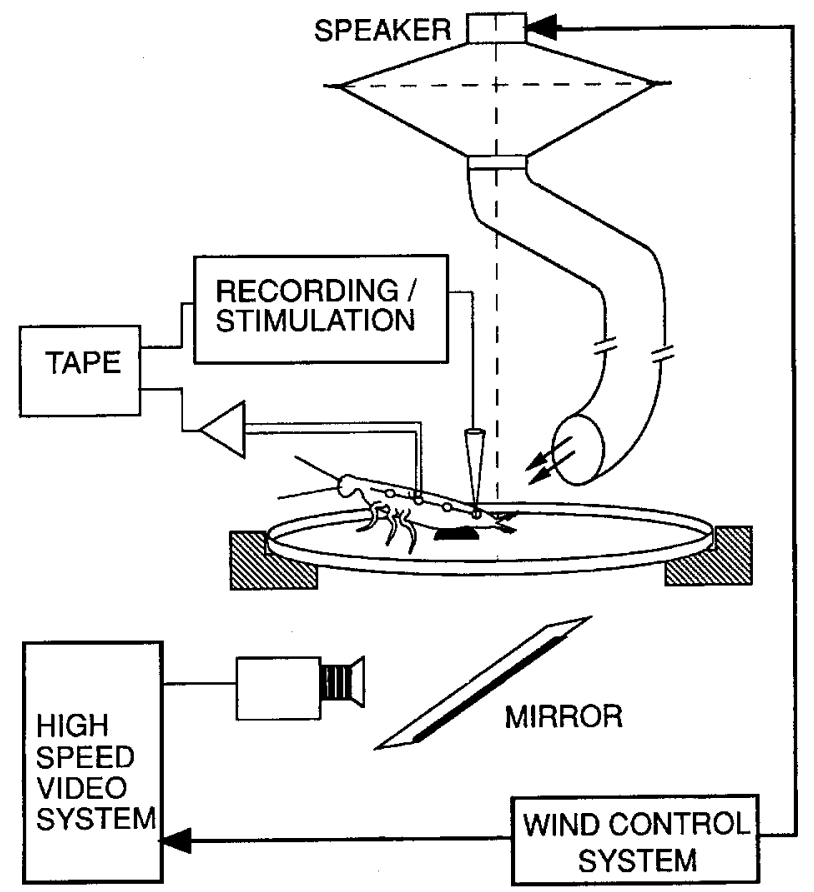

Figure 1. The experimental setup. The cockroach, fixed to a slick, transparent surface, could move its legs freely. The rotating tube from the speaker delivered wind puffs from various angles. Intracellular recording and stimulation were performed in a GI, and hook electrodes monitored extracellular activity of the nerve cord. The behavioral responses were monitored by the video through a mirror below the transparent surface.

We recorded the wind-evoked escape behaviors by a high-speed video camera (NAC, Tokyo, Japan) at 250 frames/sec. The angle of view was from the animal's ventral side, through the glass substrate. Each escape turn direction is characterized by a unique profile of leg movements (Nye and Ritzmann, 1992). These behavioral details are preserved, even in tethered cockroaches, dissected for neural recording (Camhi and Levy, 1988; Liebenthal et al., 1994). We analyzed the leg movements frame by frame on a personal computer using a video analysis program (MTV; Data Crunch, San Clemente, CA). To determine the directions of the animals' turning responses, we measured the coxa-femur (CF) joint angle of the different legs, one frame before an escape response began and again three frames $(12 \mathrm{msec})$ later. Subtracting the first angle from the second yielded the CF joint movement for each leg.

In some experiments, it was necessary to determine the relative strength of a left turn, away from a right wind source. For this, we developed a measure that uses the profile of changes in CF joint angle from all six legs (see Results). In other experiments, it was necessary to determine the cockroach's tendency to turn left versus right. For this, we analyzed the CF joints of only the front and middle pairs of legs (because the angle difference between the joint movements of two rear legs was practically zero), and used the profile of the $\mathrm{CF}$ angle changes in these four legs.

The wind receptor cells excite a group of identified GIs whose axons ascend from the last abdominal ganglion of the CNS in which they receive their sensory input from the cerci, through the thoracic ganglia in which they excite motor circuits to the legs. Three pairs (left and right GIs 1, 2, and 3) are especially important for establishing the escape turn direction (Comer, 1985; Comer et al., 1988; Liebenthal et al., 1994; Levi and Camhi, 1995). Each GI has a unique directional response to wind, including a unique best excitatory direction (BED) (Kolton and Camhi, 1995). To record from and stimulate the axons of these GIs intracellularly, in the posterior abdominal region, we used glass microelectrodes with an impedance range of 20-40 $\mathrm{M} \Omega$ (Liebenthal et al., 1994). The electrodes were back filled with $6 \%$ carboxy fluorescein and filled with 3 $\mathrm{M} \mathrm{KCl}$. At the end of each experiment, we delivered $100 \mathrm{nA}$ hyperpolarizing current for 10-20 min to the GI and identified it, on the basis of its cell body position and dendritic tree shape, in a whole mount using a fluorescence microscope (Daley et al., 1981). The GIs show no synaptic interconnections in the last abdominal ganglion (Mizrahi and Libersat, 1997). Thus, adding spikes to one GI does not alter the spike trains that ascend in other GIs from this ganglion to the thorax.

For intracellular stimulation, we used the discontinuous current-clamp mode of the Axoclamp 2B amplifier (Axon Instruments, Foster City, CA). This method enabled us to administer short pulses of up to $100 \mathrm{nA}$ and still record ongoing and evoked spikes with the same electrode (Fig. 1; see Fig. 4B). (Some of this current may have leaked from the axon, because the cockroach's running movements caused some decrease in the quality of the electrode penetration.) We also recorded extracellular activity of the whole nerve cord with a pair of silver hooks positioned under the abdominal nerve cord, amplified with an AC amplifier (Grass P15; Grass Instruments, Quincy, MA), for indication of the condition of the cord and the verification of conduction of the intracellularly evoked spikes. We stored all physiological data on videotapes using a Neurocorder (Neuro Data, New York, NY) for later analysis on a personal computer program (Computerscope; RC Electronics, Santa Barbara, CA).

\section{RESULTS}

\section{Measuring left-turning tendency}

We first developed a means to evaluate the strength of the cockroach's turning tendency to the left, in response to a wind stimulus from the right. This was needed to determine the behavioral effect of an intracellularly injected train of spikes in a GI. We delivered wind stimuli to each of 20 animals that were tethered on the lubricated glass substrate but were not dissected. The stimuli were presented at $20^{\circ}$ intervals, from $10^{\circ} \mathrm{R}$ to $150^{\circ} \mathrm{R}$, in a randomized sequence. We videotaped the leg movements and measured the CF joint movement of each leg, as described in Materials and Methods.

Figure 2 plots the joint angle changes for each of the six legs in response to wind from different directions. For all the legs except $\mathrm{R} 1$ (the right front leg), the angle change increased as the wind direction was changed from near 0 (head end) to large angles (rear end). There were, however, individual differences. Our measurements somewhat underestimated the movements of the $\mathrm{CF}$ joints of the front legs, because these front legs are held at an angle oblique to the view of the camera.

We transformed these data into a single parameter that could describe turn direction. For this, we combined the data from all six legs into a single linear relationship between wind angle and the movements of the joints, using a multiple linear model (statistical program JMP-SAS for MacIntosh computers). This model performed a least-square optimization among the six variables (six leg joints) and the wind direction. The least-square error method determined the coefficients that would best relate the combinations of leg movements to the wind direction.

We also incorporated into the model the parameter of the stationary angle of each joint before the onset of the turn, because the initial position of the leg has been shown to contribute to determining its movement response to wind (Camhi and Levy, 1988). For each of the six legs, we divided the data pool into three categories: (1) trials in which the stationary CF angle was within \pm SD of the mean for all trials; (2) trials in which this angle was more than SD above this mean; and (3) trials in which this angle was more than SD below this mean. In each trial, we calculated separately the coefficient for each leg, according to which of the three categories it belonged. It was these three sets of coefficients that we used in the model, according to the initial leg position on each trial.

This model provided a measure we call left-turning tendency, whose range extends from 0 (the left-turning tendency extrapolated to a wind angle of $180^{\circ}$ ) to 1 (extrapolated to $0^{\circ}$ ) (Fig. $3 A$ ). The left-turning tendency varied linearly with wind angle $(p<$ 


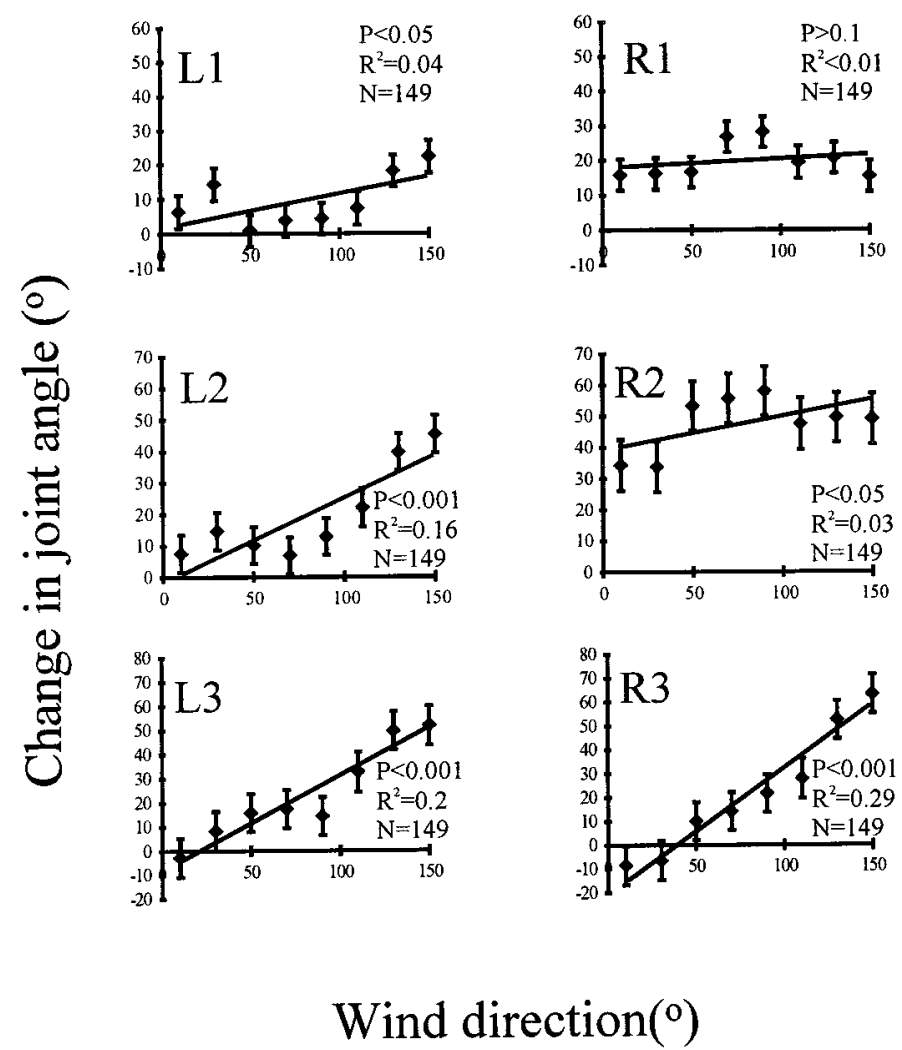

Figure 2. Changes of the CF joint angles as a function of wind direction. Stimuli ranged from near head-on wind (i.e., $\sim 0^{\circ}$ ) to wind from close to behind $\left(150^{\circ}\right)$. Each plot shows the means \pm SEM from one leg: left front (L1), left middle ( $L 2)$, left hind (L3), and the same for the right legs. All correlations are significant except R1.

$\left.0.01 ; R^{2}=0.34\right)$. Importantly, when we superimposed on this graph data from the control trials of experimental animals (i.e., trials of dissected cockroaches with the electrodes in place but with no spike injection), these points were not significantly different from those of the nondissected cockroaches for wind from the same direction $\left(30^{\circ}\right.$ and $\left.90^{\circ}\right)$ or the closest tested direction $\left(120^{\circ}\right)$ (Fig. $3 A$ ). This justified the use of left-turning tendency to evaluate turns in the experimental trials.

\section{Electrical stimulation of right GI3}

To study the effect of changing neural activity on the sizes of wind-evoked turns, we chose to focus on a single neuron, namely GI3 (specifically, the right GI3, ipsilateral to the right wind stimulus). This choice was based on this cell having the narrowest receptive field of any of the GIs 1, 2, and 3. Thus, even by delivering wind from $90^{\circ} \mathrm{R}$, which is not very far away from the BED of the cell $\left(30^{\circ} \mathrm{R}\right)$, this GI would be excited relatively little by wind, permitting us to make a substantial addition to its spike train by the electrical stimulation.

We presented the trials in pairs: a control trial without electrical stimulation, either followed by or preceded by the experimental trial with electrical stimulation. (The control trial came first in half of the trials, and the experimental trial came first in the other half.) In the control trial in Figure $4 A$, the wind puff evoked five spikes from its onset until a criterion time we set of $4 \mathrm{msec}$ before the movement response of the legs; this is approximately the time needed for a given GI spike to be processed and influence the movement of the leg (Camhi and Nolen, 1981). The first spike

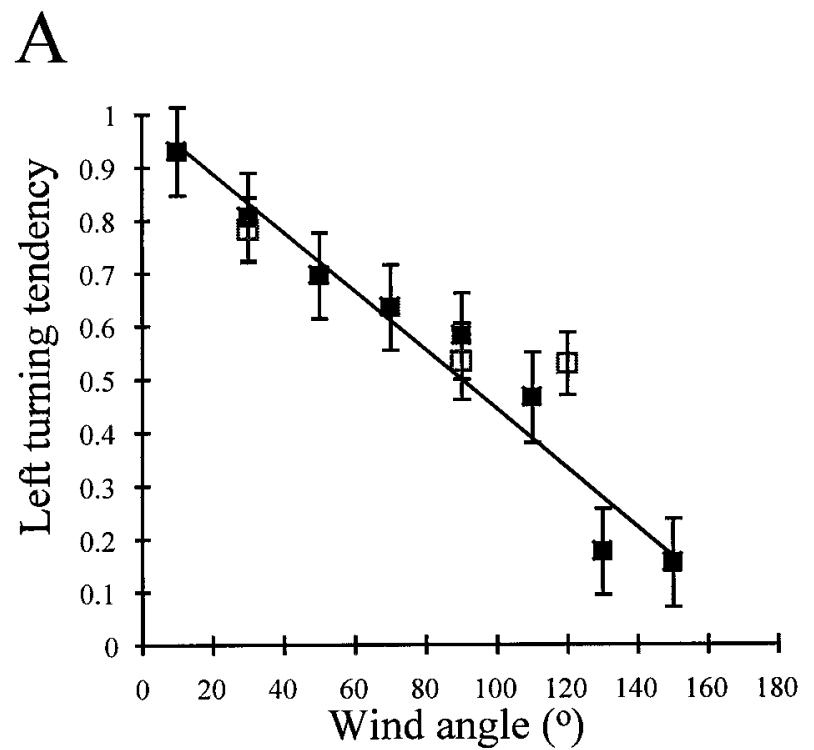

$\mathrm{B}$

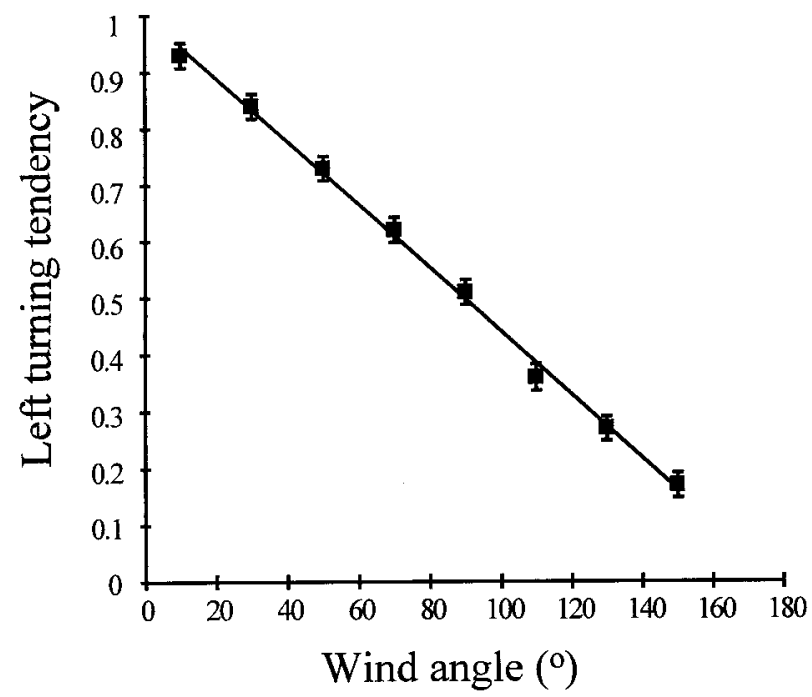

Figure 3. Left-turning tendency as a function of wind direction. A, Filled symbols, Data from 20 tethered, but not dissected, cockroaches. Open symbols, Data at three wind angles from experimental, dissected cockroaches. Means \pm SEM shown. $B$, Based on the same data as the filled symbols in $A$, but here the linear regression was calculated separately for each animal (mean \pm SEM).

evoked in the impaled GI occurred at $7 \mathrm{msec}$ after a timing signal used to activate the wind system. The hook recording (Fig. 4A, middle trace) shows the spikes of all the GIs and numerous other cells excited by the wind stimulus.

In the immediately following experimental trial (Fig. 4B), we delivered a train of electrical pulses at $400 \mathrm{~Hz}$, beginning at the time of the wind onset signal and continuing until after the turn had begun. For the first two electrically evoked spikes and for some of the subsequent ones, the associated spike can be seen clearly in the hook recording. This indicates that the GI3 spike was indeed conducted anteriorly from the site of the microelectrode in the axon of the GI. In this trial, the number of spikes recorded in GI3 from wind onset to the criterion time doubled to 10 spikes (Fig. 4B, top trace).

This same pattern of just one control and one experimental 

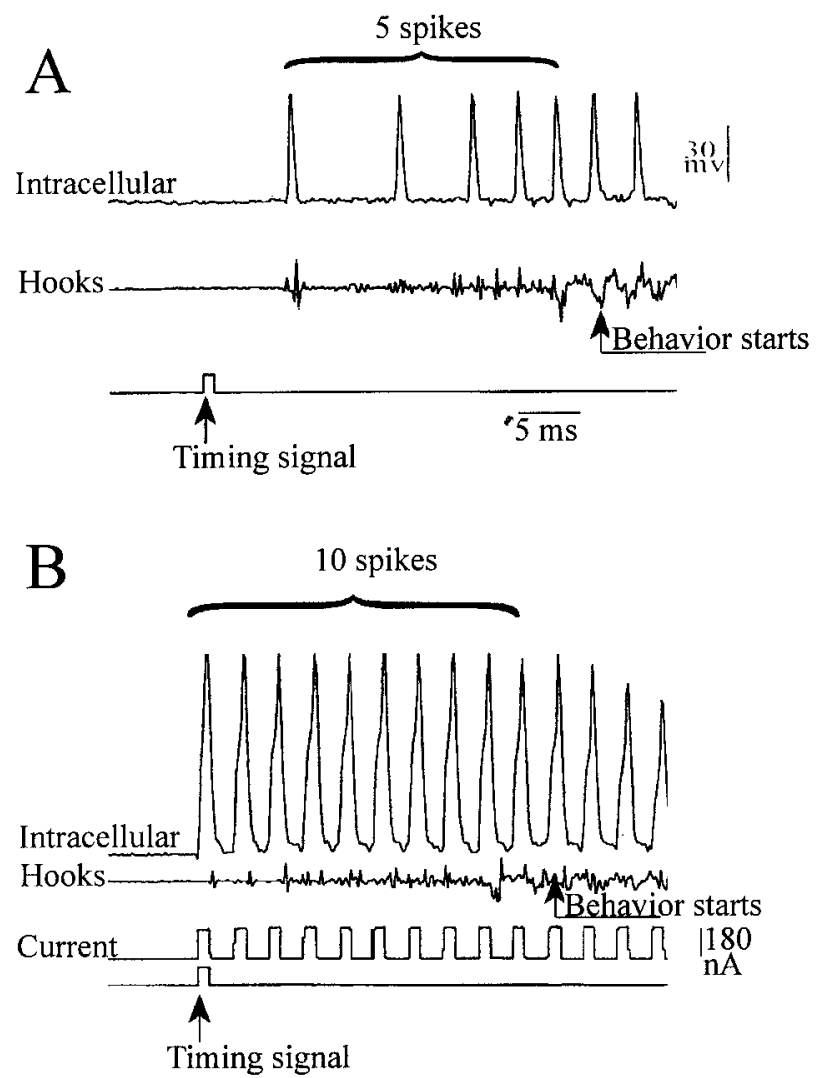

Figure 4. Physiological recording and stimulation of the right GI3 from a sample experiment. $A$, Wind alone, from $90^{\circ}$ right, with no electrical stimulation. Top trace, GI3 response to the wind. Five spikes were evoked before the criterion time, as explained in Materials and Methods. Middle trace, Nerve cord response to the wind. Bottom trace, Timing signal to the speaker that later delivered the wind puff to the cerci. $B$, Same as $A$, except for the addition of the electrical stimulus train (next to bottom trace) that evoked a spike train in GI3 (top trace). In this trial, 10 spikes occurred in GI3 before the criterion time. Calibration for top trace in $A$ also applies to top trace in $B$.

trial for each animal was used on each of the 17 animals; thus 17 control and 17 experimental trials comprised the data set for this experiment. We used a range of stimulus frequencies, from 330 to $500 \mathrm{~Hz}$, according to the ability of each cell to follow the stimulus train, which correlated generally with the quality of the electrode penetration. The mean number of added spikes in all 17 experiments was 4.7.

In analyzing the results, we compared the cockroach's leftturning tendency on each experimental trial with that of the associated control trial. The electrical stimulation altered the left-turning tendency toward a larger turn $(p<0.01$; Wilcoxson paired test) (Fig. 5), which was expected in response to wind close to the right front. This suggests that the electrical stimulation had caused a change in the cockroach's perception of the wind direction, from the actual direction $\left(90^{\circ} \mathrm{R}\right)$ toward the BED of the stimulated right $\mathrm{GI} 3\left(30^{\circ} \mathrm{R}\right)$.

The injection of these $330-500 \mathrm{~Hz}$ spike trains caused a mean increase of 0.09 in the left-turning tendency. If one omits the one extreme datum at -1 , this mean increase becomes 0.16 . From Figure $3 A$, one can calculate that this 0.16 increase is $58 \%$ of the difference in left-turning tendency to wind stimuli alone from $90^{\circ}$ $\mathrm{R}$ (the actual wind direction) and $30^{\circ} \mathrm{R}$ (the BED of right GI3). This $58 \%$ of $60^{\circ}$ gives $34.8^{\circ}$ as the effect of the added spikes. That

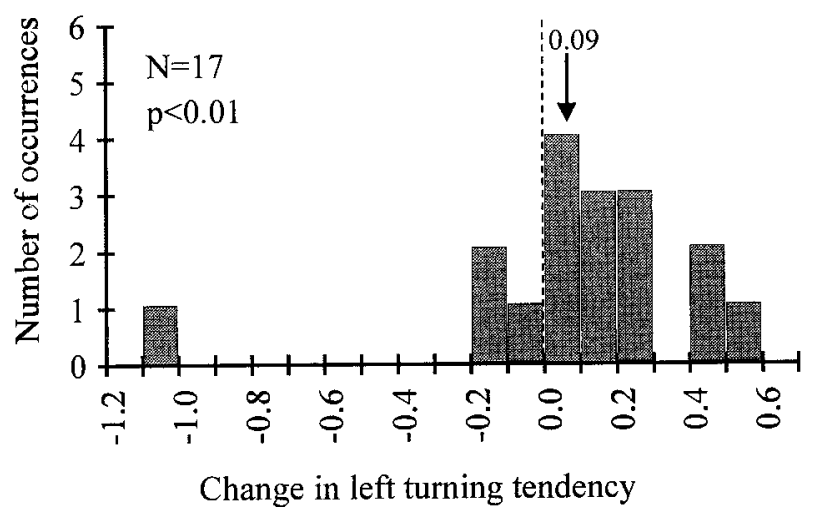

Figure 5. Distribution of changes in left-turning tendency resulting from electrical stimulation to GI3. The change is represented as the difference between the single control trial and the single experimental trial for each of the 17 different animals. Positive values indicate enhanced turning tendency caused by the stimulation. Arrow points to the mean of all data.

is, the cockroach perceived the wind as coming from $34.8^{\circ}$ more anterior than it actually did, and this caused the change in turn direction. Dividing 34.8 by the mean of 4.7 spikes that we added gives a rough estimate of $7.4^{\circ}$ of change in perception of wind angle per added spike.

\section{Effect of varying spike frequency}

We used the measure of left-turning tendency to test for a winner-take-all mechanism. To do this, we generated in GI3 spike trains of different frequencies and determined whether the shift of left-turning tendency was proportional to the spike frequency or increased abruptly in an all-or-none manner. Thus, we repeated the same experiment as in Figures 4 and 5, but this time we changed the frequency of the electrical pulses. We also changed the wind direction from $90^{\circ} \mathrm{R}$ to $130^{\circ} \mathrm{R}$, to provide a broad range of possible left-turning tendencies over which the shift could take place. In this experiment, we gave, on each of 14 animals, three control trials (wind only), plus generally two trials at each of five stimulation frequencies between 50 and $550 \mathrm{~Hz}$. We analyzed only experiments whose regression line, like that in Figure $6 A$, showed an upward slope with an $R^{2} \geq 0.2$ (10 of the 14 animals tested).

Figure $6 A$ shows the results from a single animal in which the left-turning tendency appears to have increased proportionately to the frequency of injected spikes $\left(p<0.01 ; R^{2}=0.7\right.$; Pearson correlation). To examine on one graph the data from all 10 animals analyzed, we normalized the 10 graphs by setting the mean value of all points on a given graph equal to the mean of all points for each other graph. The result is seen in Figure $7 B$, which, like $7 A$, appears to show a proportional increase in leftturning tendency with frequency of GI spikes. (On this graph, the negative values indicate those less than the means, and the units on the ordinate represent SDs.)

There exists, however, the possibility that the regression line of Figure $7 B$ results from two sets of points (a low set in the left half of the graph and a high set on the right half) rather than a continuous increase. This would indeed be consistent with a winner-take-all mechanism. To test this more rigorously, we performed a linear regression for each animal separately and analyzed the residual of the regression. (For each individual experiment, the residual is the distance of each point from the regression line.) If there were two sets of points, one low and the other high, one would expect the residual to vary as shown in 

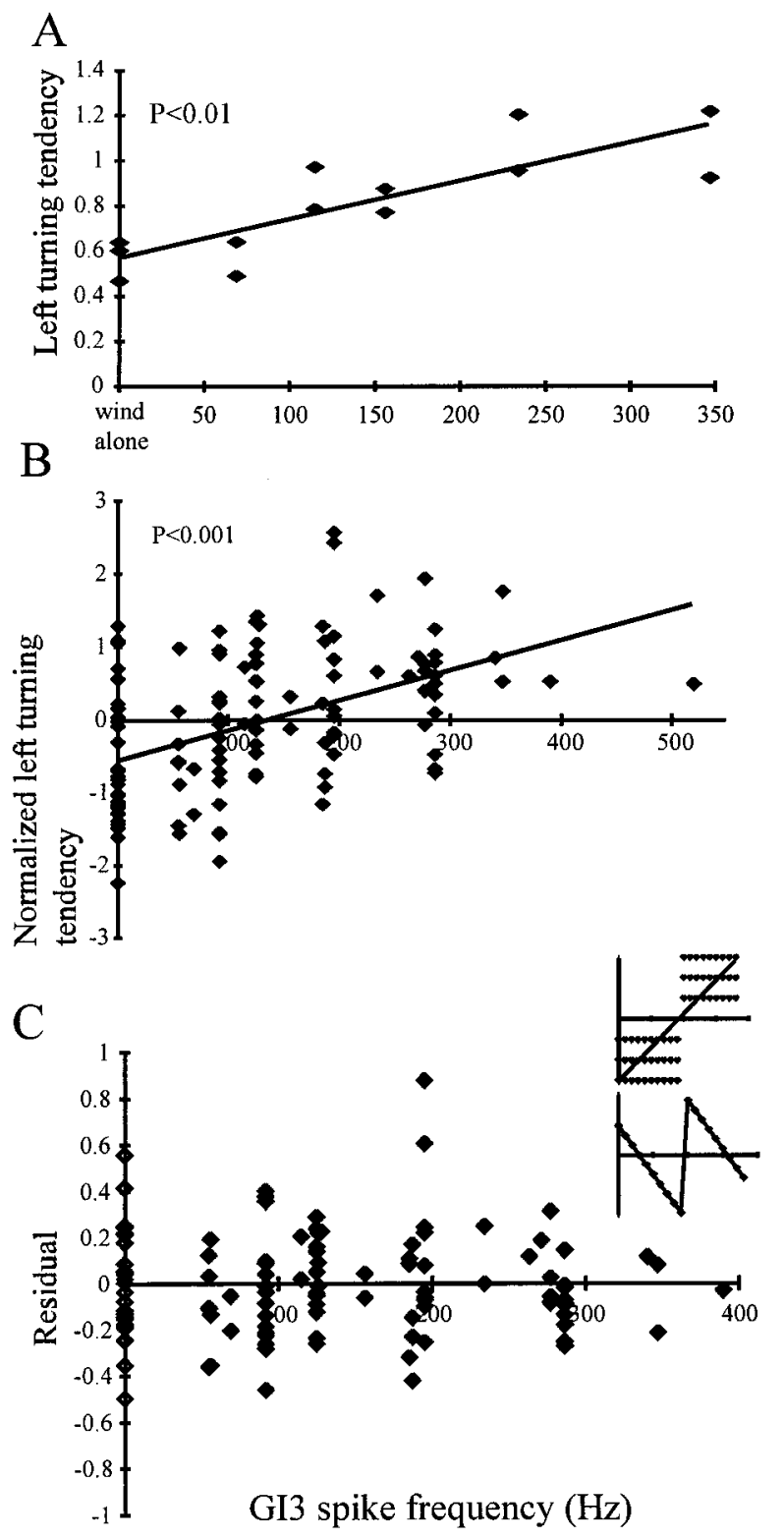

Figure 6. Correlation between spike frequency in right GI3 and the left-turning tendency. $A$, Sample experiment and its linear regression line. Wind stimulation alone was presented three times, whereas each of five spike frequencies was presented twice. $B$, Normalized, pooled results from all the animals tested and the regression line. $C$, The residual values of individual regressions as a function of the spike frequency. Inset, Top graph, Theoretical bimodal distribution of left-turning tendencies for different GI3 spike frequencies. Bottom graph, The residual expected from these data. Notice that, on the large graph of the actual data, the vertical spread of the data points is fairly consistent for different spike frequencies and not of the saw-tooth pattern shown in the bottom inset graph.

the inset of Figure $7 C$, with large negative and positive peaks near the middle of the frequency range. This was clearly not the case; rather, the residual magnitude was fairly consistent for all of the GI3 spike frequencies (Fig. 7C).

The critical analysis, however, is to determine whether the values of the normalized left-turning tendency (Fig. 6B) at intermediate GI3 spike frequencies are significantly different from those at both lower and higher GI3 spike frequencies. To test this, we divided the GI3 spike frequencies into three bins: (1) controls (wind only, average spike frequency of $57 \mathrm{~Hz} ; 66 \%$ of the trials had zero or one spike); (2) spike frequencies between 55 and 200 $\mathrm{Hz}$; and (3) spike frequencies between 200 and $520 \mathrm{~Hz}$. The normalized left-turning tendencies for these three bins are shown in Figure $7 A$. Indeed, the middle group is significantly different from each of the two extreme groups. We further ruled out that the intermediate category consisted of two distinct data groups; Figure $7 B$ shows that the distribution of these data are unimodal, and it is not different from a normal distribution $(p>0.2$; Kolmogorov-Smirnov test for normality).

Thus, there appears to be not a single step, from small left turns evoked by the wind to large left turns evoked by the wind plus electrical stimulation; rather, there is a staged transition. Presumably, with sufficient additional data points, additional significantly different, intermediate angles could be found. However, a single intermediate category already rules out a winner-takeall mechanisms.

\section{Behavioral intermediates and angular resolution}

Although testing for a winner-take-all mechanism requires physiological methods such as those reported above, behavioral observations can provide supportive evidence. Especially in relatively simple systems, involving relatively few neurons, a winnertake-all system might produce discrete jumps in the measure of behavior: one jump for each participating cell. Examining Figure $3 A$, no such discrete jumps appear; rather, there is a progressive decrease in left-turning tendency.

Nevertheless, it was useful to determine the behavioral resolution more accurately. Specifically, if the cockroach were able to resolve behaviorally many different wind angles, producing a corresponding number of different turn directions, this would hint that the cockroach's small number of GIs would be an insufficient number to operate on a winner-take-all basis; there simply would be not enough potential "winning" cells for all the different turn sizes.

To measure the behavioral discrimination of wind angle, it was preferable to reduce the behavioral variation among animals. We did this by multiple linear regression, as in Figure $3 A$; however, in the present analysis, we calculated the coefficients separately for each animal and then proceeded as in Figure $3 A$.

In the resulting graph $\left(p<0.001 ; R^{2}=0.84\right)$ (Fig. $\left.3 B\right)$, differences in wind direction as small as $20^{\circ}$ are statistically significant $\left(p<0.01\right.$ for wind directions between $30^{\circ}$ and $150^{\circ}$, except $110^{\circ}-130^{\circ}$; Student's $t$ test). Thus, the directional resolution of the escape turn is at least as low as $20^{\circ}$. Therefore, if a winner-take-all mechanism were to operate, there would need to be a minimum of approximately eight different cells with different BEDs, each $20^{\circ}$ apart, ranging from $20^{\circ} \mathrm{R}$ to $160^{\circ} \mathrm{R}$, and a corresponding set of approximately eight left cells. Although we do not know all of the neurons that take part in the escape behavior, at least among the GIs that are thought to initiate the turn, there are insufficient neurons to explain the mechanisms on a winner-take-all basis.

\section{Electrical stimulation of left Gls 1, 2, and 3}

Although a winner-take-all mechanism appears not to be involved in determining the sizes of left turns, such a mechanism could determine whether a turn will be to the left or the right. To test this, we injected spike trains into left GIs while giving right wind. It is known that injecting such spike trains can flip the turn direction from right to left (Liebenthal et al., 1994). Does this flip occur in an all-or-nothing (i.e., winner-take-all) manner or gradually with increasing frequencies of injected spikes? 


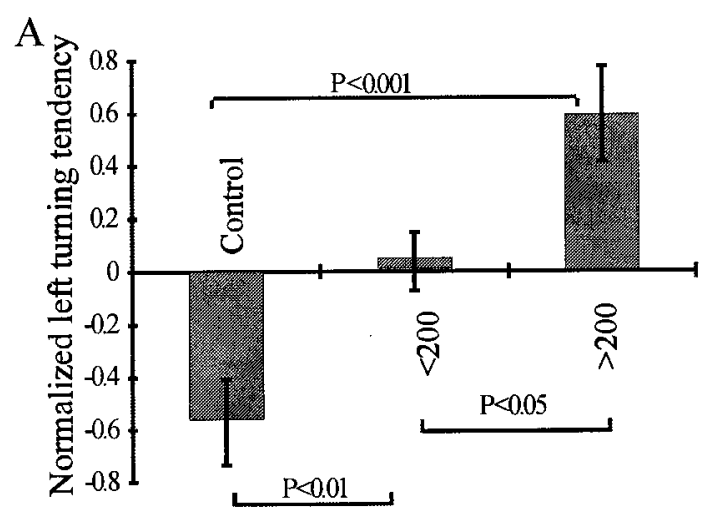

GI3 spike frequency $(\mathrm{Hz})$
B

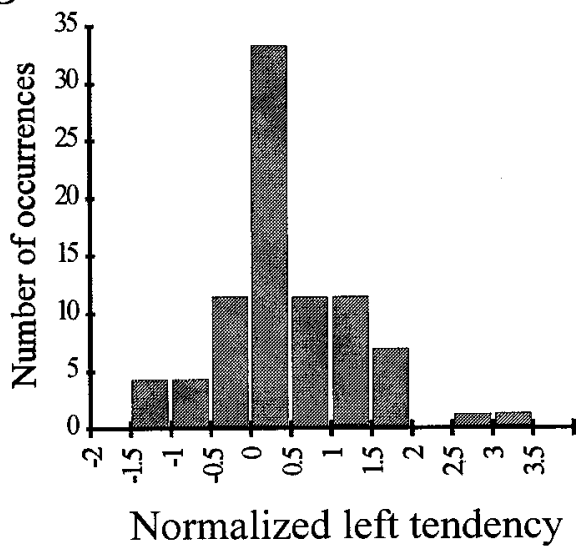

Figure 7. The effect of three different ranges of electrical stimulation of the right GI3 on left-turning tendency. $A$, Control (no electrical stimulation) and two different frequency ranges all give responses significantly different from one another. ( $p$ values shown are Tukey's multiple comparisons test on each of the categories.) $B$, Distribution of the normalized turning tendency in the middle category $(<200 \mathrm{~Hz})$ of $A$.
We delivered spike trains of increasing frequencies into the left GIs 1,2 , or 3 , together with wind puffs from $90^{\circ}$ right. We analyzed the difference in the CF joint movements of the left versus the right front and middle legs. [In general, for both of these pairs of legs, the CF joint ipsilateral to the wind source opens more than the contralateral joint (Fig. 2).]

For this analysis, we subtracted the CF joint movement of the left leg from that of its right partner for each trial. This gave the left-right angle difference for each leg pair for each trial. We then normalized the change in joint angle for all of the trials of each animal as follows. We subtracted the mean angle difference of all trials from the CF angle change of each trial. Then, we divided the result by the SD of all trials. In 12 experiments on as many cockroaches (four each using GI1, GI2, and GI3), we gave three trials each of wind only, at 100, 300, and $400 \mathrm{~Hz}$ stimulation, in a randomized sequence.

The added spike trains significantly altered the left-rightturning tendency for GIs 1,2 , and 3 ( $p<0.01,0.05$, and 0.01, respectively; Pearson correlation). In all three cases, the direction of the effect was the same: the injected spikes directing the turn more toward the right, that is, toward rather than away from the side of the wind stimulus. Normalizing these data so as to minimize interanimal differences reveals the effects of the electrical stimulation (Fig. 8A). These data confirm and extend an earlier study (Liebenthal et al., 1994) by showing that this effect occurs regularly for each of the left GIs 1,2 , and 3 .

For all these GIs, the left-right-turning tendency was significantly altered by electrical stimulation $(p<0.05$; Pearson correlation). A significant switch from left to right turns occurred for GIs 1 and 3 ( $p<0.01$; signed rank test), whereas for GI2, significant right turning was never achieved, the turning tendency for $400 \mathrm{~Hz}$ stimulation being not significantly different from 0 . As Figure $8 A$ shows, for all three GIs, the change was gradual and correlated with the frequency of the injected spike train. That is, intermediate spike train frequencies evoked a behavior that, according to our measure of left-right-turning tendency, was neither a left nor a right turn. Our data did not reveal whether these latter behaviors may represent a straight forward run (as occurs in response to wind from behind; $180^{\circ}$ ) or perhaps a backward movement (as often occurs in response to wind from the front; $0^{\circ}$ ) (Nye and Ritzmann, 1992).

Because high-frequency electrical stimulation to GIs 1 and 3 switched the escape behavior from left to right turns, one can measure the turning tendency of these reversed escapes. (We did this by simply switching the left and right legs in the analysis and calculating left-turning tendency, as above.) In the trials involving left GI1, without electrical stimulation, the left-turning tendency was 0.40 , and with maximal stimulation the right turning tendency was 0.24 . (These are raw, not normalized, values.) These turning tendencies correspond to turns in response to winds from $107^{\circ}$ right and $135^{\circ}$ left, respectively (Fig. $3 A$ ). In the trials involving GI3, without electrical stimulation the left-turning tendency was 0.33 , and with maximal stimulation the right turning tendency was 0.73. These numbers correspond to turns in response to wind from $100^{\circ}$ right and $48^{\circ}$ left, respectively. Thus, stimulating the left GI3 whose BED is in the left front quadrant evoked a large turn, like that normally produced by left front wind.

It remained possible that the data of Figure $8 A$ consist of either clear left turns or clear right turns and that the proportion of these two categories varied with spike injection frequency. Such a situation could still be interpreted as involving a winner-take-all mechanism. We analyzed the regression residual to test this possibility. For this, to reduce scatter and thus reveal more clearly any trends, we used the raw, unnormalized data only of the legs that showed a significant effect of the electrical stimulation. If there were only clear left and clear right turns, varying in their proportion, one would expect the residual to be greatest in intermediate range of injected spike frequencies. This was not the case (Fig. 8B).

\section{DISCUSSION}

The present study attempts to discern the type of information processing that occurs in the neural pathway between the cockroach GIs and the motor response of escape. Rather than attempting to work out the entire neural circuit to attain this goal, we have used judicious manipulation of the information code in the GIs together with careful behavioral analysis to test a specific hypothesis as to how the information is processed. This has revealed that a winner-take-all mechanism is not involved in the determination of direction by the GIs.

Our principle evidence against a winner-take-all mechanism is that, with the wind stimulus delivered from an angle distant from a given BED of a GI, adding increasing numbers of spikes to that GI produces a proportional, rather than a precipitous, shift in the direction of the escape turn. In the experiments involving electrical stimulation of right GI3 and with the wind coming from the right side posterior to the $\mathrm{BED}$ of this GI, we interpret the result as follows. The more spikes we added to this GI, the more this cell promotes the perception of wind from near the front right, rather 


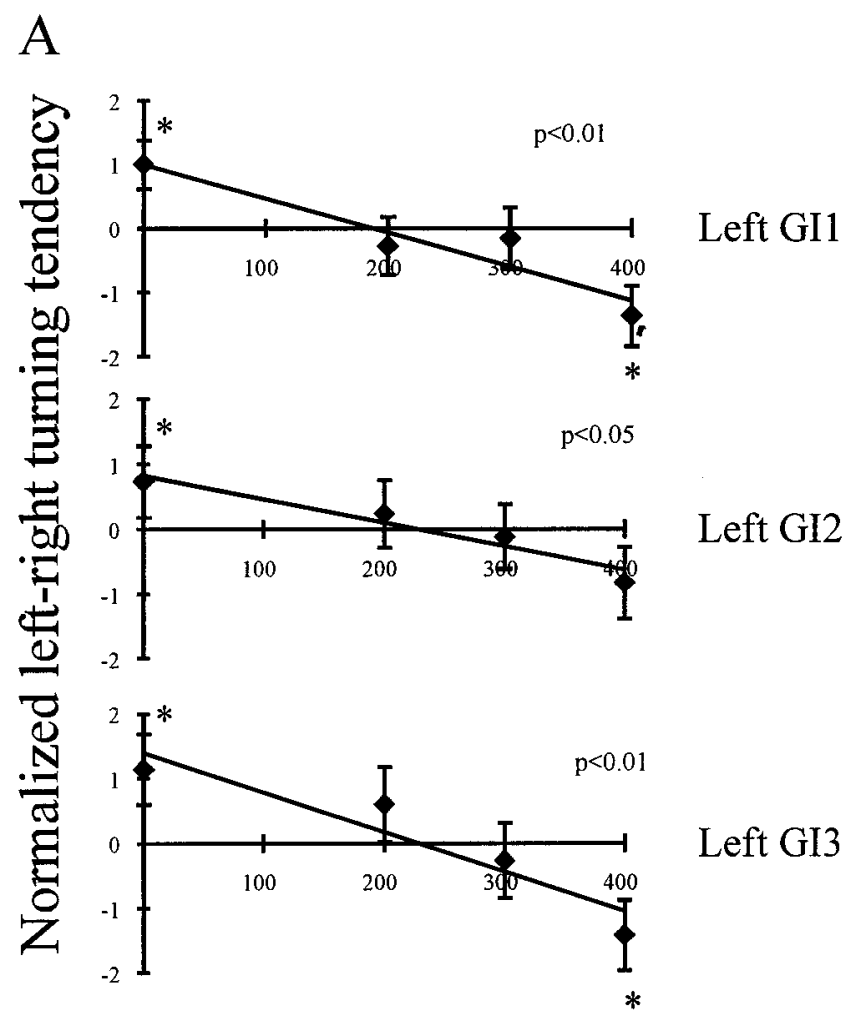

\section{Spike frequency $(\mathrm{Hz})$}

B

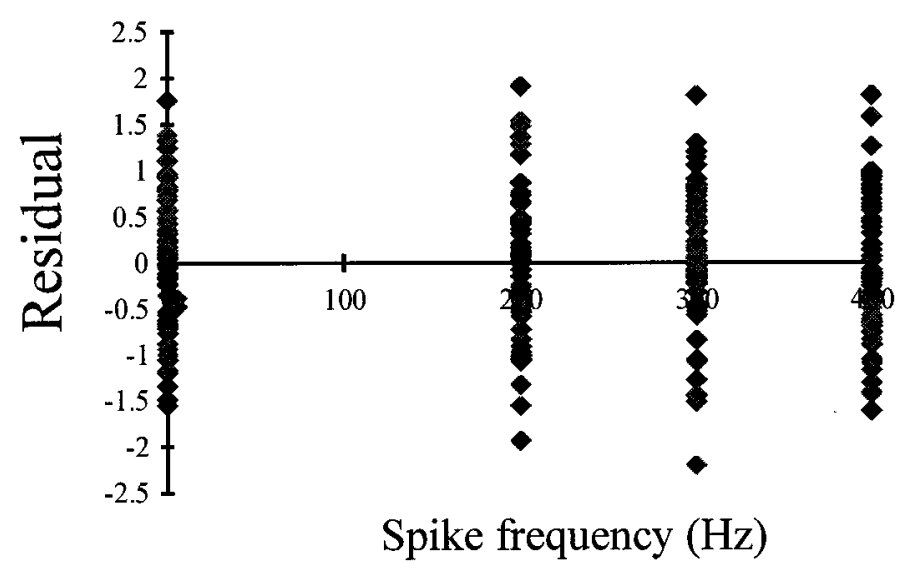

Figure 8. Effect of electrical stimulation of left GIs 1, 2, and 3 on the directions of turns in response to wind from $90^{\circ}$ right. $A$, Mean \pm SEM responses to wind alone (leftmost point on each graph) and three different GI spike frequencies. The left-turning tendency is normalized and therefore dimensionless. $B$, The residual values of the individual regressions from $A$, as a function of GI spike frequency. The residuals for all three graphs in $A$ are plotted here together. Notice that the vertical spread of the points is quite consistent for all different spike frequencies.

than the rear right; thus, the more it produces a large left turn, reflected in our measure of enhanced left-turning tendency.

The interpretation is similar, although a bit more complex, in the experiments performed with right wind stimulation and spike addition in a left GI. Again, the result is a gradual effect. Interestingly, when stimulating the left GI3, the right turns evoked by maximal stimulation were large right turns, not small ones. This indeed is as expected, given the frontal BED of the stimulated GI3. This implies then that the cockroach's perception of wind direction was drawn across the frontal midline by the electrical stimulation, from right front, to midline, to left front.

But how can one understand the very small responses seen in this experiment with intermediate stimulus frequencies (Fig. $8 A)$ ? In earlier observations of the escape directions of freely running cockroaches, in response to wind stimuli from this narrow range of frontal angles, it appeared that the behavior had an all-or-nothing quality. Most stimuli from just right of the midline gave large left turns, and most from just the left of the midline gave large right turns (Camhi and Tom, 1978). This indeed had hinted at the possibility of a winner-take-all mechanism.

More recent observations have indicated that there are intermediate response variants. A neural model of the cockroach escape system predicts, as is seen in Figure $8 A$, that increasing the activity of the GIs contralateral to the wind would produce escapes that are neither left nor right turns, and still stronger contralateral activity would switch the turn direction (Ezrachi et al., 1999). Behavioral studies have shown that, as the wind angle is brought very close to the midline in front of the cockroach, the initial response is often a backward movement that then grades into a sharp turn away from the side of the wind (Nye and Ritzmann, 1992). It is possible that the very low values of turning tendency for intermediate stimulus frequencies of left GI3 in Figure $8 \mathrm{~A}$ reflect this initial backward movement, captured on video in our $12 \mathrm{msec}$ sample time. Thus, the absence of strong left or right turns for these intermediate stimulus frequencies is consistent with the known behavioral responses to midline wind stimuli.

It is not possible, on the basis of the experiments reported here, to determine what type of neural interactions might be responsible for the observed responses to GI stimulation. However, one neural feature that appears to be ruled out is strong mutual inhibition among the outputs of the different GIs, or among separate groups of follower cells or circuits that each GI may have. Such strong mutual inhibition would have been implied by the very winner-take-all mechanism that our experiments have ruled out. However, these physiological experiments do not rule out weak mutual inhibition. For instance, if the right GI3 with its right front BED was mutually inhibitory with right GI2 with its right rear $\mathrm{BED}$, and if these inhibitions were weak, there could still be intermediate directional responses to wind from angles between these two BEDs. Thus, adding intermediate numbers of spikes to right GI3, when the wind stimulus is from the right rear, could still evoke intermediate left-turning tendencies such as those seen in Figure $7 A$.

It would be most likely, however, that any such weak mutual inhibition would be reflected in the behavioral responses to wind stimuli alone from different right angles. Specifically, one would expect a nonlinearity in the left turn sizes. However, the behavioral graphs of left-turning tendency in response to different angles of right wind is highly linear (Fig. 3). Thus, these graphs not only corroborate the absence of strong mutual inhibition but even hint at the absence of weak mutual inhibition among the GIs or their follower cells and circuits.

Our result of a $20^{\circ}$ directional resolution of the behavior is far from the $4.7-7^{\circ}$ resolution found for cricket escape behavior, using information theory methods (Theunissen and Miller, 1991). Crickets perform escape turns whose initial, prejump portion is remarkably like that of the cockroach (Tauber and Camhi, 1995). The resolution difference presumably reflects in part the differ- 
ence in the parameters measured: behavior in the cockroach versus GI responses in the cricket. Surely there are numerous sources of scatter of the directional information between the neural activity of the GI and the actual movement response. One should note, however, that unlike the information theory calculations, our observations do not give minimal resolution. Thus, a larger data sample could well reveal an improved behavioral resolution of the cockroach escape behavior.

Previous measurements of the escape response of free-ranging cockroaches have shown the turn direction to be highly variable (Camhi and Tom, 1978). The results we have obtained are strikingly different from this (Fig. 3). One reason is that, in freeranging cockroaches, the criterion for measuring the end point of the turn was the moment when two successive cine' frames showed no continuing change in body angle, typically much later than the $12 \mathrm{msec}$ sample time of the present work. In that time, many additional factors could well influence turn size, including antennal or other sensory inputs. By restricting our present measurements to the initial response, which is probably influenced primarily by the GI spikes and not other confounding neural inputs, the behavior is seen to be much more consistent from trial to trial. It is particularly striking that the cockroaches respond in this regular way, even under conditions in which the body has dissected and prepared for electrical stimulation (Fig. 3A, open symbols).

The absence of a winner-take-all mechanism suggests that the different GIs and their postsynaptic cells collaborate with each other to determine direction. In a separate paper (Levi and Camhi, 2000), we show that this collaboration involves a population vector code.

\section{REFERENCES}

Camhi JM, Levy A (1988) Organization of a complex movement: fixed and variable components of the cockroach escape behavior. J Comp Physiol [A] 163:317-328.

Camhi JM, Nolen T (1981) Properties of the escape system of cockroaches during walking. J Comp Physiol [A] 142:339-346.

Camhi JM, Tom W (1978) The escape behavior of the cockroach Periplaneta americana. I. Turning response to wind puffs. J Comp Physiol [A] 128:193-201.

Comer C (1985) Analyzing cockroach escape behavior with lesions of individual giant interneurons. Brain Res 335:342-346.

Comer CM, Dowd JP, Stubblefield GT (1988) Escape responses following elimination of the giant interneuron pathway in the cockroach, Periplaneta americana. Brain Res 445:370-375.

Daley D, Vardi N, Appignani B, Camhi J (1981) Morphology of the giant interneurons and cercal nerve projections of the American cockroach. J Comp Neurol 196:41-52.

Eaton RC, DiDomenico R, Nissanov J (1991) Role of the Mauthner cell in sensorimotor integration by the brain stem escape network. Brain Behav Evol 37:272-285.

Ezrachi E, Levi R, Camhi JM, Parnas H (1999) Right-left discrimination in a biologically oriented model of the cockroach escape system. Biol Cybern 81:89-99.

Georgopoulos AP, Schwartz AB, Kettner RE (1986) Neuronal population coding of movement direction. Science 233:1416-1419.

Gras H, Kohstall D (1998) Current injection into interneurones of the terminal ganglion modifies turning behaviour of walking crickets. J Comp Physiol [A] 182:351-361.

Groh JM, Born RT, Newsome WT (1997) How is a sensory map read out? Effects of microstimulation in visual area MT on saccades and smooth pursuit eye movements. J Neurosci 17:4312-4330.

Kolton L, Camhi JM (1995) Cartesian representation of stimulus direction: parallel processing by two sets of giant interneurons in the cockroach. J Comp Physiol [A] 176:691-702.

Krasne FB, Lee SC (1988) Response-dedicated trigger neurons as control points for behavioral actions: selective inhibition of lateral giant command neurons during feeding in crayfish. J Neurosci 8:3703-3712.

Levi R, Camhi JM (1995) Testing for a population vector code for wind direction in the cockroach giant interneurons. Soc Neurosci Abstr 20:418.

Levi R, Camhi JM (2000) Population vector coding by the giant interneurons of the cockroach. J Neurosci, 20:3822-3829.

Lewis JE, Kristan WB (1998) Quantitative analysis of a directed behavior in the medicinal leech: implications for organizing motor output. J Neurosci 18:1571-1582.

Liebenthal E, Uhlmann O, Camhi JM (1994) Critical parameters of the spike trains in a cell assembly: coding of turn direction by the giant interneurons of the cockroach. J Comp Physiol [A] 174:281-296.

Mizrahi A, Libersat F (1997) Independent coding of wind direction in cockroach giant interneurons. J Neurophysiol 78:2655-2661.

Nye SW, Ritzmann RE (1992) Motion analysis of leg joints associated with escape turns of the cockroach, Periplaneta americana. J Comp Physiol [A] 171:183-194.

Roberts A (1968) Recurrent inhibition in the giant-fibre system of the crayfish and its effect on the excitability of the escape response. J Exp Biol 48:545-567.

Rose GJ, Kawasaki M, Heiligenberg W (1988) "Recognition units" at the top of a neuronal hierarchy? Prepacemaker neurons in Eigenmannia code the sign of frequency differences unambiguously. J Comp Physiol [A] 162:759-772.

Salzman CD, Newsome WT (1994) Neural mechanisms for forming a perceptual decision. Science 264:231-237.

Sparks DL, Holland R, Guthrie BL (1976) Size and distribution of movement fields in the monkey superior colliculus. Brain Res 113:21-34.

Tauber E, Camhi JM (1995) The wind-evoked escape behavior of the cricket Gryllus bimaculatus: integration of behavioral elements. J Exp Biol 198:1895-1907.

Theunissen FE, Miller JP (1991) Representation of sensory information in the cricket cercal sensory system. II. Information theoretic calculation of system accuracy and optimal tuning-curve widths of four primary interneurons. J Neurophysiol 66:1690-1703.

Wine J, Krasne FB (1972) The organization of the escape behavior in the crayfish. J Exp Biol 56:1-18. 